\title{
A method of curvature mode difference and wavelet transform to damage detection on space grid structure
}

\author{
Li-Mei Zhang ${ }^{1, a}$, Xiang-kun Yang ${ }^{2}$ and Wei-ran Liu ${ }^{1}$ \\ ${ }^{1}$ School of Civil Engineering, Hebei University of Science and Technology, Shijiazhuang, \\ Hebei Province, 050018, China \\ ${ }^{2}$ NORENDAR International, Shijiazhuang, Hebei Province, China
}

\begin{abstract}
A method combined curvature mode difference and wavelet transformation was used to determine the damage location of space gird structure. This method considered the curvature mode difference of pre and post damage as the wavelet transform analysis signal. Moreover, these signals were analyzed by wavelet transform using wavelet function $\mathrm{db} 3$. As the wavelet transform coefficient occur sudden change, the damage locations of links will be obtained. In this paper, square pyramid space grid structure, orthogonal space truss structure, three way latticed grid and honeycomb triangular pyramid grid structure will be analyzed using this method to identify single damage and multi-damage location. The solutions from numerical simulations show that this method can take effective identification of single damage location and multi-damage location of square pyramid space grid structure by the first mode condition. And this method has versatility to detect damages from different space grid structures.
\end{abstract}

\section{Introduction}

Curvature mode (Li 2002, Chen 2007, Ding 2007, VALSAN 2008) is one of the mode parameters, and curvature mode often expressed the signals of dynamics testing. But the signals of curvature mode are continuous, and the influence of noise must be considered. So, these signals are not very sensitive to structural damage. However, the method of wavelet transformation can show the local features of signals and remove noise. Therefore the wavelet transformation was used to discrete these continuous curvature mode signals and eliminate noise. According to these features, the wavelet transformation can be used to detect structural damages (Law 2005, Zhang 2006, Yan 2012). Sun Zengshou (2005) use the method of curvature mode and wavelet transform to identify damage, considered the difference of wavelet transform coefficient of pre and post damage as injury index; this method also was used by Yin Zhixiang (2009)to detect the damage location of spatial latticed structure; Xu Wenjie(2008) used CWT and DWT wavelet mode to test the damage location of spatial latticed structure; Zhang Yue(2010) use the method of wavelet analysis and curvature mode to detect damage of square pyramid grid structure.

Through analysis, the curvature modes difference from pre and post damage were considered as importing signals which were analyzed by db3 wavelet in this paper. This method was used to detect

\footnotetext{
${ }^{\mathrm{a}}$ Corresponding author : zhanglimei168@126.com
} 
the damage location of square pyramid grid structure. The solutions show that this method can locate single and multi damage location more accurately.

\section{Theory of damage detection}

\subsection{Theory of curvature mode}

Link damage often occur in local structure, which can change structural local parameter, for example stiffness EI, structural section area, etc.. The change of EI can cause curvature mode change and these changes from pre and post damage can express the damage index to detect damage location(Wang 2012).

Structure vibration equation is written as:

$$
M \ddot{v}+C \dot{v}+K v=f(t)
$$

While, $M, C, K$ respectively represent matrix of mass, damp and stiffness, $v$ is vibration displacement, $f(t)$ is the structural exciting load. According to mode theory, the solution of Eq.(1) can be expressed a s:

$$
v=\sum_{i=1}^{n} \varphi_{i} q_{i}(t)=\Phi q
$$

While, $\phi_{i}$ is displacement modal vibration shape and $q_{i}(t)$ is modal coordinates, $\Phi$ is modal matrix.

Curvature modes are often obtained by difference approximate calculation and can be written as:

$$
\varphi_{i}^{\prime \prime}=\left(\varphi_{i-1}-2 \varphi_{i}+\varphi_{i+1}\right) /\left(l_{i-1, i} l_{i, i+1}\right)
$$

While, subscript $i$ express the $i$ th measuring point; $l_{i-1, i}$ express distance between measuring point $i$ 1 and $i$. Then pre and post damage of curvature mode difference $\Delta \phi_{k i}^{\prime \prime}$ can be expressed as:

$$
\Delta \varphi_{k i}^{\prime \prime}=\varphi_{k i}^{\prime \prime}-\varphi_{k i}^{\prime \prime d}
$$

While, $k$ express the kth mode; $\Delta \phi_{k i}^{\prime \prime}$ is curvature mode difference of node $i$ with the $k$ th mode; $\phi_{k i}^{\prime \prime d}$ is curvature mode difference of node $i$ after structural damage; $\phi_{k i}^{\prime \prime}$ is curvature mode difference of node $i$ with intact structure.

\subsection{Theory of wavelet transformation}

The purpose of wavelet transformation is to analysis the response signals by wavelet function. Through analysis, some mutation information was obtained and these information can determine the situation of this structure. In this paper, the curvature mode difference $\Delta \phi_{k i}^{\prime \prime}$ was considered as input signal $S$. These signals were analyzed by specific wavelet function $\varphi(t)$ which have two basic parameters scale $a$ and translation distance $b$. Considering structural discrete characteristics, the wavelet transform coefficients $C$ adopt discrete wavelet transform (DWT). Through analysis, the wavelet transform coefficients $C$ will be gained and this coefficient will be written as follows:

$$
C=\left(W_{\phi} S\right)(a, b)=\left\langle S, \phi_{a, b}\right\rangle=\frac{1}{\sqrt{a}} \int_{-\infty}^{+\infty} S \phi\left[\frac{t-b}{a}\right] d t, \quad a>0
$$


While, $C=\left(W_{\varphi} S\right)(a, b)$ is wavelet transform coefficient (Zhang 2012), “ \langle\rangle ” means inner product. This equation show that the minimal changes of wavelet function $\varphi(t)$ can lead to larger variety of wavelet coefficient $C$, signal $S$ is variable that change by damage. Therefore, the bigger the wavelet transform coefficient $C$ means the larger the mutation degree of signal, namely damage location. So, with different wavelet function $\varphi(t)$, the analyzing signals will show huge difference. The db3 wavelet belongs to Daubechies group was selected in this paper because of its properties of fast transformation, better orthogonality and vanishing moment(Li 2012).

\section{Numerical simulations of four type space grid structures}

\subsection{Model and damage conditions of four type space grid structures}

Element link8 was selected to simulate bars in software ANSYS. Its elastic modulus $E=2.06 e 11 P a$, poisson ratio $\mu=0.3$, section area of upper and lower chord is $0.00005 \mathrm{~m}^{2}$, web member is 0.000025 $\mathrm{m}^{2}$, this truss bearing uniform load $1 \mathrm{kN} / \mathrm{m}^{2}$, using surrounding support and constraint in bottom chord nodes. Square pyramid space grid truss shows in figure 1(unit $\mathrm{mm}$ )and this structure has 326 nodes, 1200 bars. Orthogonal space truss structure shows in figure 2 (unit $\mathrm{mm}$ )and it has 348 nodes, 1139 bars. Three way latticed grid structure shows in figure 3 (unit mm)and it has 656 nodes, 3096 bars. Honeycomb triangular pyramid grid structure shows in figure 4(unit $\mathrm{mm}$ ) and it has 390 nodes, 1110 bars. Damage degree and damage conditions list in table 1. Single damage and multi damages all exit. Damage express link element damage and damage was obtained by the reduction of section area. The damage degree of upper chord was 10\%, 30\% and 50\% respectively and other damage degree all considered $10 \%$.

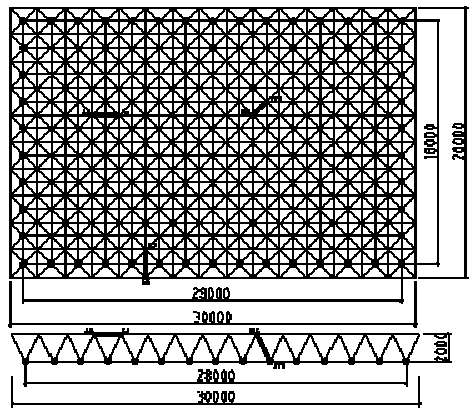

Figure 1. Square pyramid space grid structure.

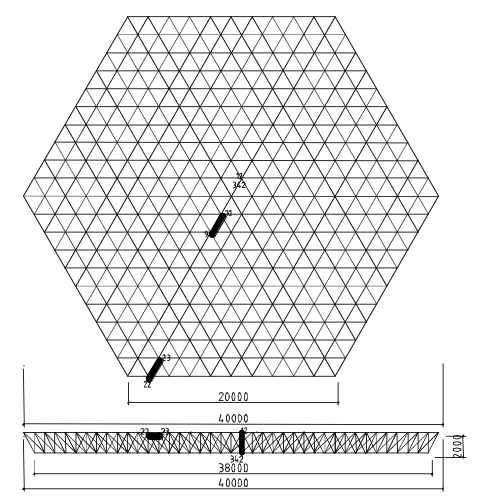

Figure 3. Three way latticed grid structure.

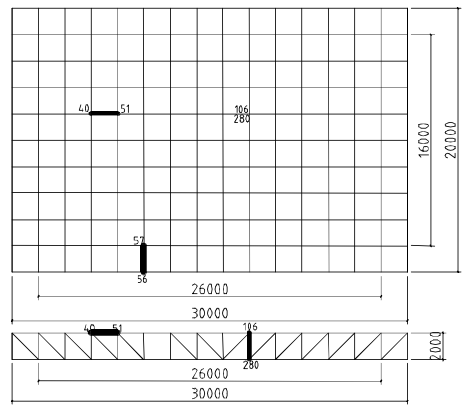

Figure 2. Orthogonal space grid structure.

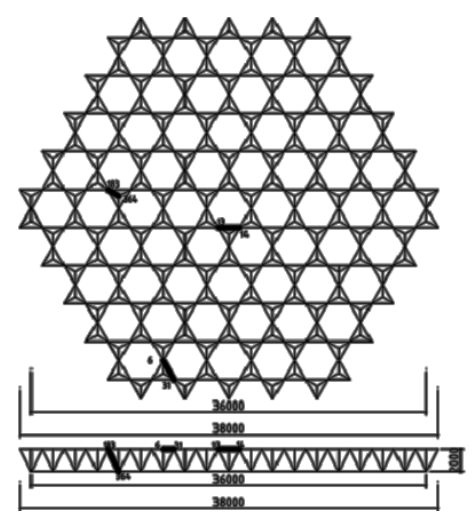

Figure 4. Honeycomb triangular pyramid grid structure. 
Table 1. Damage conditions of four type space grid structures.

\begin{tabular}{|c|c|c|c|c|}
\hline Structure type & Damage Condition & Damage Member (Node Number) & Damage Degree & Member Type \\
\hline \multirow{6}{*}{$\begin{array}{l}\text { square pyramid } \\
\text { space grid } \\
\text { structure }\end{array}$} & 1 & $51(56-57)$ & $10 \%$ & Upper Chord \\
\hline & 2 & $51(56-57)$ & $30 \%$ & Upper Chord \\
\hline & 3 & $51(56-57)$ & $50 \%$ & Upper Chord \\
\hline & 4 & $985(106-273)$ & $10 \%$ & Web Chord \\
\hline & 5 & $200(40-51)$ & $10 \%$ & Middle Mhord \\
\hline & 6 & $51 \cdot 200 \cdot 985$ & $10 \%$ & - \\
\hline \multirow{6}{*}{$\begin{array}{l}\text { orthogonal space } \\
\text { truss structure }\end{array}$} & 1 & $51(56-57)$ & $10 \%$ & Upper Chord \\
\hline & 2 & $51(56-57)$ & $30 \%$ & Upper Chord \\
\hline & 3 & $51(56-57)$ & $50 \%$ & Upper Chord \\
\hline & 4 & $746(106-280)$ & $10 \%$ & Web Chord \\
\hline & 5 & $200(40-51)$ & $10 \%$ & Middle Mhord \\
\hline & 6 & $51 \cdot 200 \cdot 746$ & $10 \%$ & - \\
\hline \multirow{6}{*}{$\begin{array}{l}\text { three way latticed } \\
\text { grid } \\
\text { structure }\end{array}$} & 1 & $21(22-23)$ & $10 \%$ & Upper Chord \\
\hline & 2 & $21(22-23)$ & $30 \%$ & Upper Chord \\
\hline & 3 & $21(22-23)$ & $50 \%$ & Upper Chord \\
\hline & 4 & $9(9-10)$ & $10 \%$ & Middle Mhord \\
\hline & 5 & $1853(12-342)$ & $10 \%$ & Web Chord \\
\hline & 6 & $9 \cdot 21 \cdot 1853$ & $10 \%$ & - \\
\hline \multirow{6}{*}{$\begin{array}{l}\text { honeycomb } \\
\text { triangular pyramid } \\
\text { grid structure }\end{array}$} & 1 & $82(31-6)$ & $10 \%$ & Upper Chord \\
\hline & 2 & $82(31-6)$ & $30 \%$ & Upper Chord \\
\hline & 3 & $82(31-6)$ & $50 \%$ & Upper Chord \\
\hline & 4 & $9(13-14)$ & $10 \%$ & Middle Chord \\
\hline & 5 & $1065(183-364)$ & $10 \%$ & Web Chord \\
\hline & 6 & $9 \cdot 82 \cdot 1065$ & $10 \%$ & - \\
\hline
\end{tabular}

\subsection{DWT coefficient of four type space grid structures}

Calculating the first-order curvature mode difference of above condition, then the first scale DWT coefficients of wavelet db3 were obtained and the results were shown in figure 5 to figure 8 .

From Fig.5 to fig. 8 can be seen, (1)Wavelet transform coefficients (DWT) were sensitive to the square pyramid space grid structure, orthogonal space truss structure and three way latticed grid structure, but the honeycomb triangular pyramid grid structure has poor sensitivity to DWT coefficient. (2)More great mutations in node of damage member with single damage condition and multi-damage condition were illustrated in these figures. Based on these mutations, the damages can be determined and single damage can be judged easily. (3) As damage degree increase, the value of DWT coefficient also increase, so that the size of damage degree can be pre-judgment. (4) This method is closely related to the damage location. Damage on web chord and upper chord near edge are sensitive to DWT coefficient, while damage on middle chord in the structure was insensitive relatively.

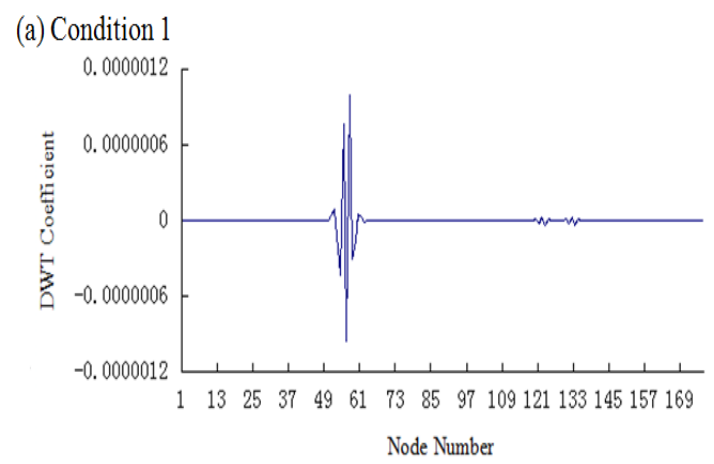

(b) Condition 2

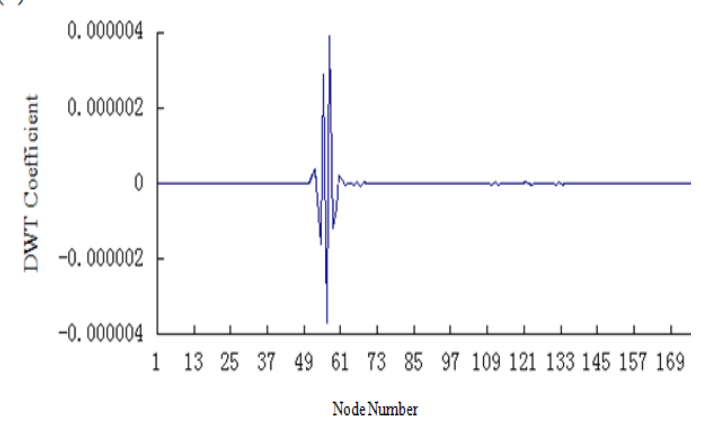



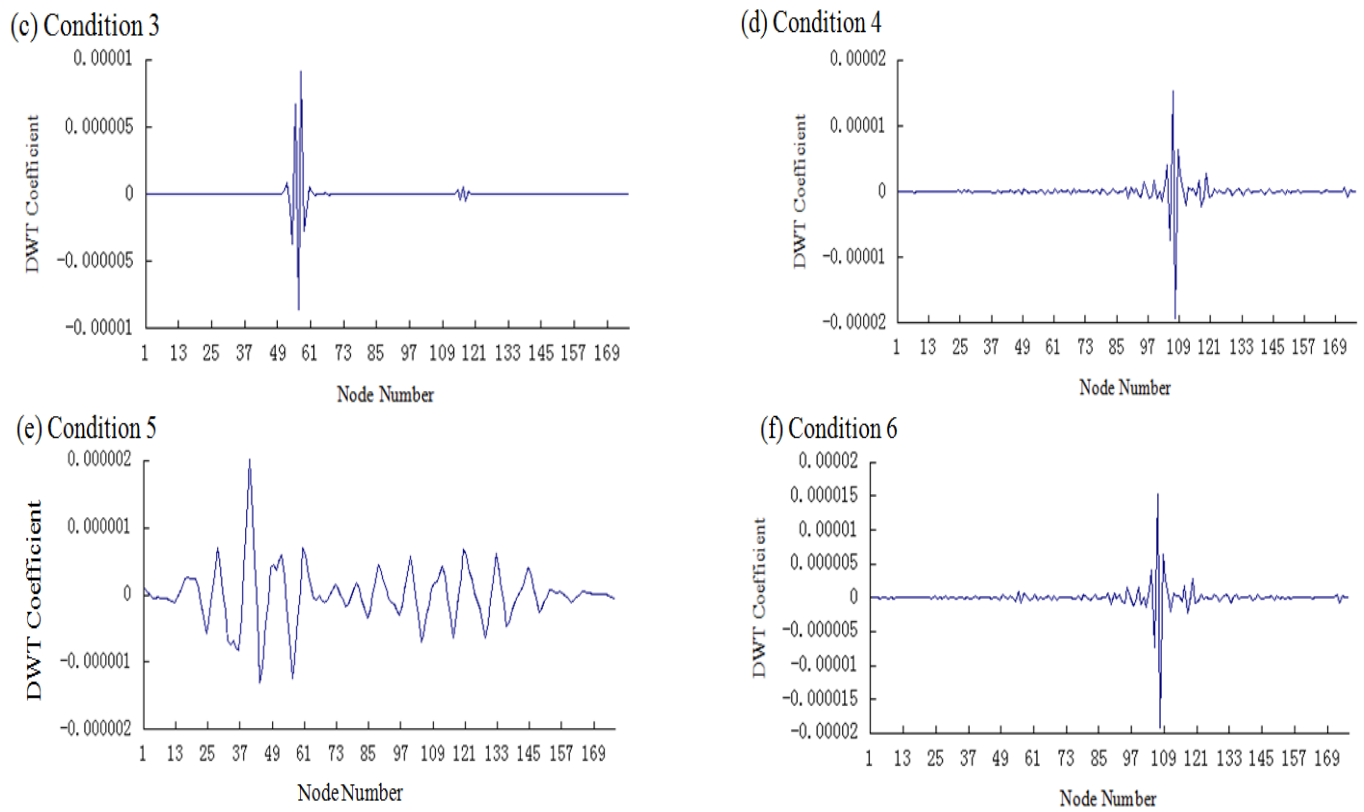

Figure 5. DWT Coefficients of square pyramid space grid structure.
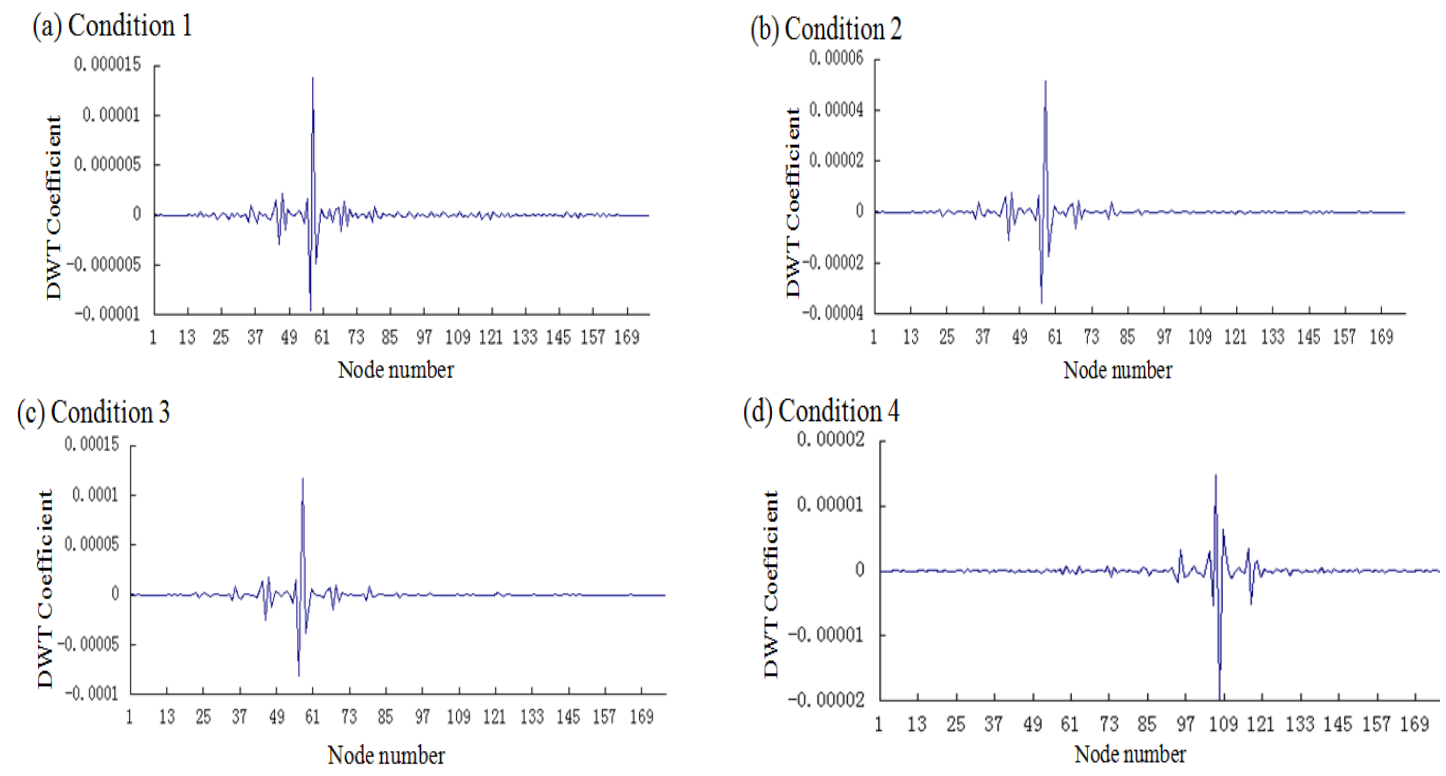

(e) Condition 5
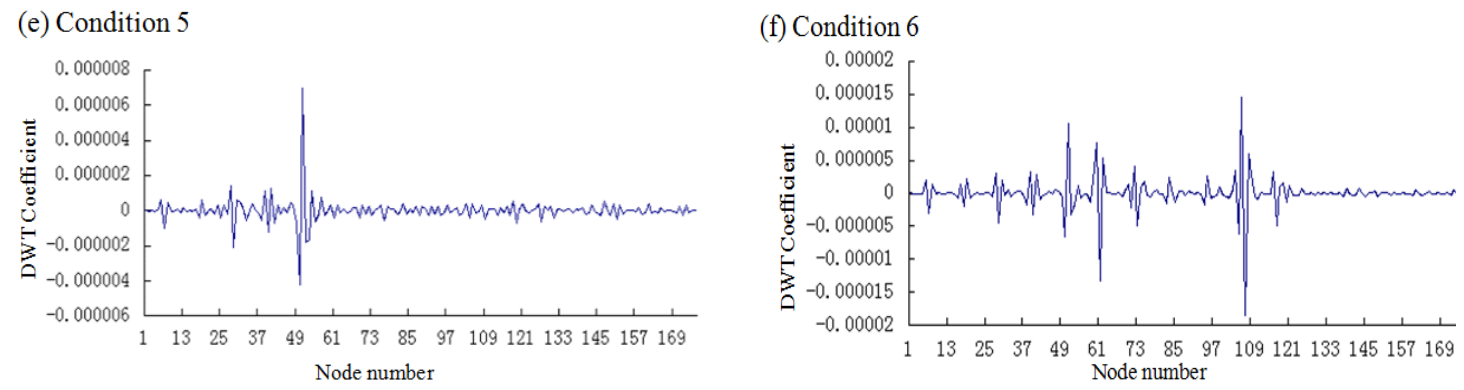

Figure 6. DWT Coefficients of orthogonal space truss structure. 


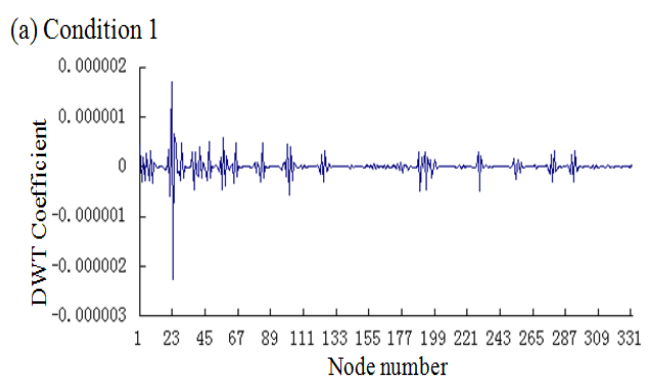

(c) Condition 3
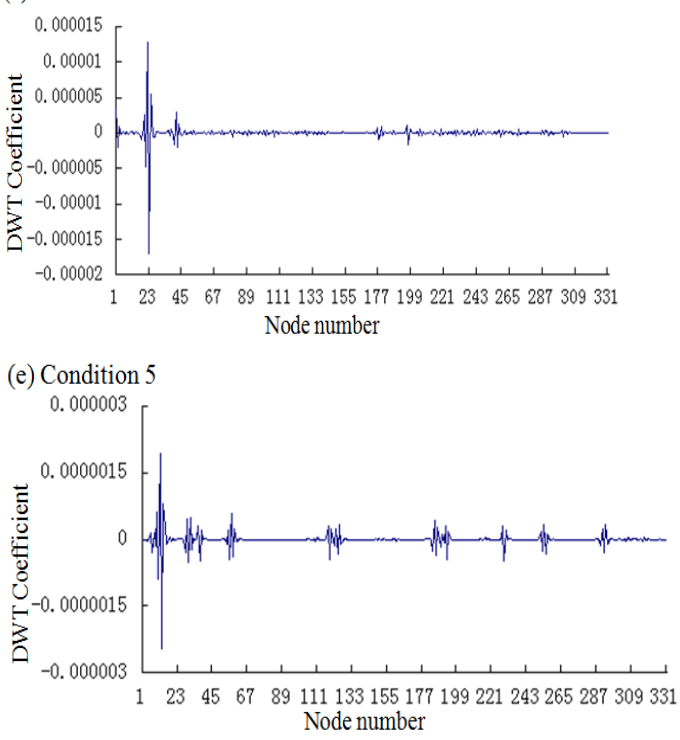

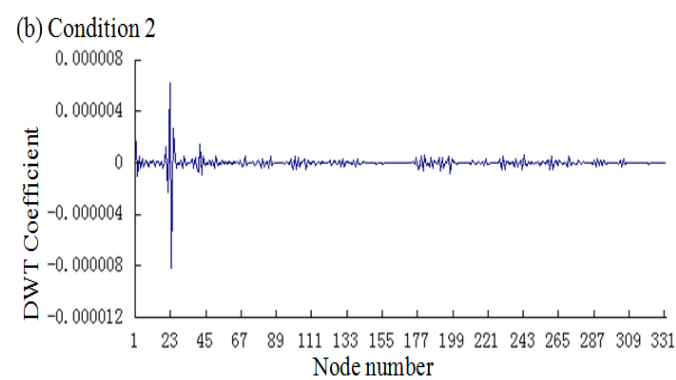

(d) Condition 4

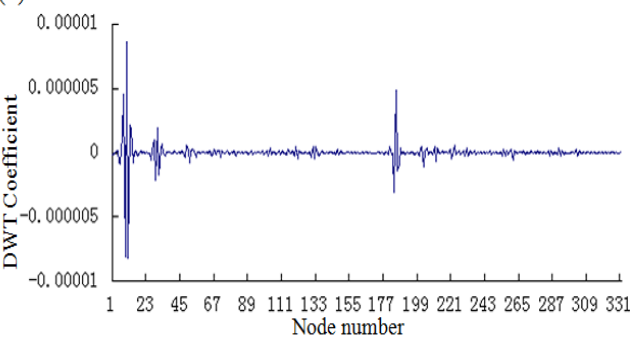

(f) Condition 6

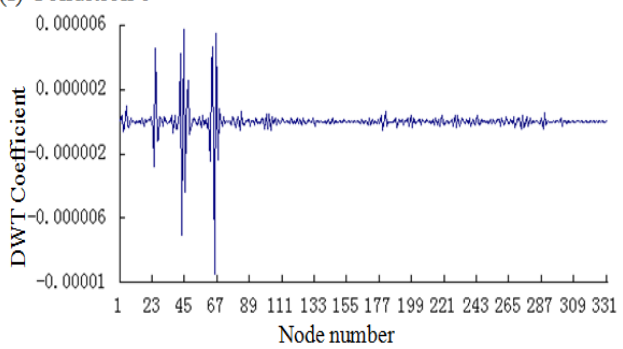

Figure 7. DWT Coefficients of three way latticed grid structure.

(a) Condition 1

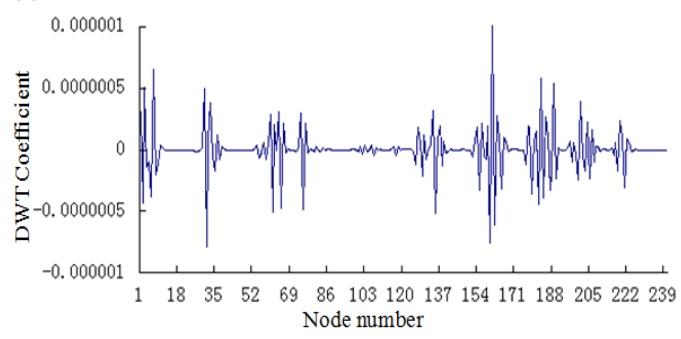

(c) Condition 3

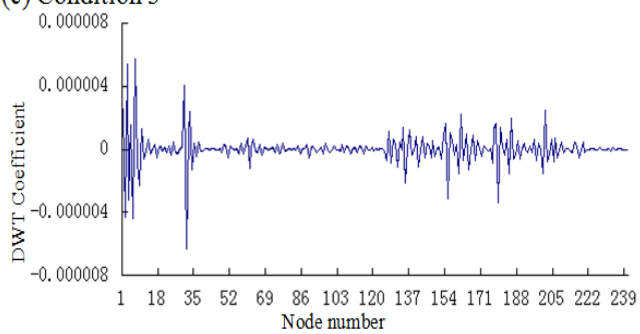

(b) Condition 2

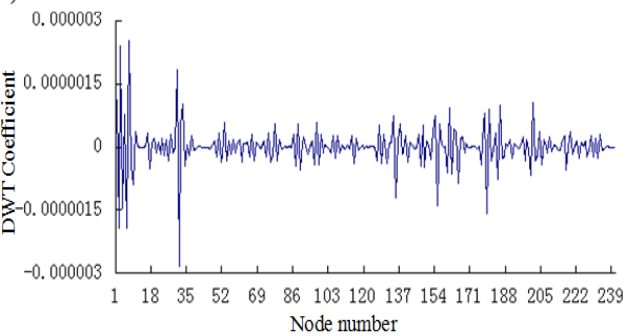

(d) Condition 4

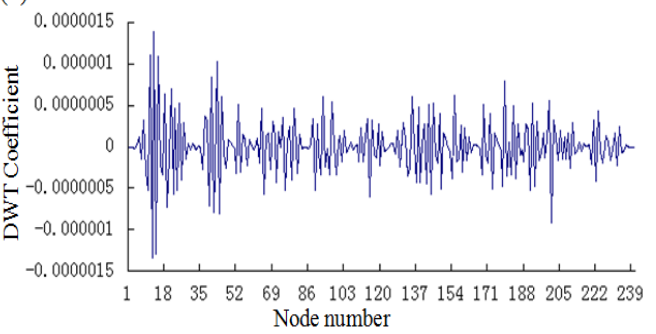



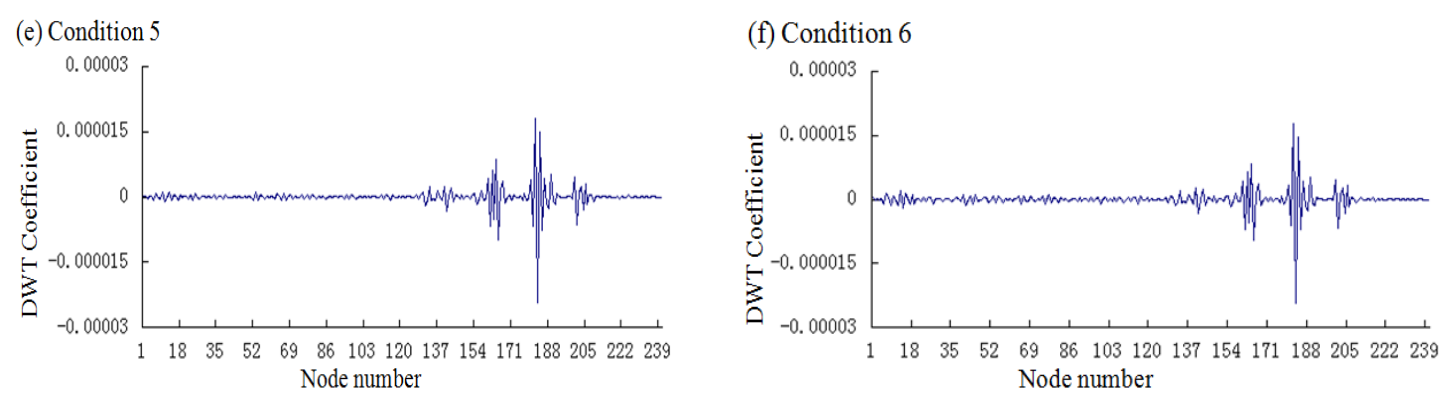

Figure 8. DWT Coefficients of honeycomb triangular pyramid grid structure.

\section{Conclusion}

This paper use a method that based on curvature mode difference and wavelet transform to judge damage location of four space grid structures. Damage expressed by reducing member elastic modulus and damage members including chord and web member, the structural curvature mode differences of pre and post damage were considered as signal which were used to do DWT by db3 wavelet. And the nodes corresponding to the maximum wavelet transform coefficient response represent damage location. The results of numerical simulation show that:

(1) The db3 wavelet transform coefficients of curvature mode difference from first mode shape of pre and post damage were used to judge damage location of square pyramid space grid structure. Through analysis, this method can obtain better identification effect in both single damage and multi-damage condition of four different space grid structures.

(2) Based on the DWT mutations, the damages' location can be determined. Damage on web chord and upper chord near edge are sensitive to DWT coefficient, while damage on middle chord was insensitive. So the links in different location have different identification results.

(3) The damage detection results show that for the same link with different damage degree, the greater degree of injury, the greater mutation value in damage node.

\section{Acknowledgement}

This work appreciates very much the Natural Science Foundation of Hebei Province (No. 2014208135) and Hebei province science and technology support project (No. 15275429).

\section{References}

1. G.Y. Li and H.W. Zheng, Measurement \& Diagnosis, Journal of Vibration, 22, 136 (2002)

2. J. Chen and F. Xiong, Journal of Wuhan University of Technology, 29, 99 (2007)

3. Y. Ding, Y. Song, and Z.X. Li, Journal of Tianjin University, 40, 726 (2007)

4. S.P. Valsan and K.S. Swarup, Electric Power Systems Research, 78, 547 (2008)

5. S.S. Law, X.Y. Li, and X.Q. Zhu, Engineering Structures, 27, 1339 (2005)

6. X.B. Zhang. Shanxi Architecture, 32, 58(2006)

7. P. Yan, H.S. Li, and J.P. Ge, Journal of Vibration and Shock, 31, 121 (2012)

8. Z.S. Sun, J.G. Han, and W.X. Ren, Earthquake Engineering and Engineering Vibration, 25, 44 (2005)

9. Z.X. Yin, W.J. Xu, and L.Q. Mao, Earthquake Engineering and Engineering Vibration, 29, 164 (2009)

10. W.J. Xu, Thesis for master degree of Liaoning Technical University (to be published, 2008) 
11. Y. Zhang, Thesis for master degree of Hebei University of science and technology (to be published, 2010)

12. Z. Wang, Sichuan Building Science, 38, 97 (2012)

13. D.F. Zhang, China Machine Press, (to be published, 2012)

14. Y.M. Li, B. Zhou, and W.J. Zhang, Spatial Structures, 18, 24 (2012) 